\title{
Nonvolatile memory effect based on gold nanoparticles doped ferroelectric liquid crystal
}

\author{
J. Prakash, ${ }^{1,2}$ A. Choudhary, ${ }^{1}$ A. Kumar, ${ }^{1}$ D. S. Mehta, ${ }^{2}$ and A. M. Biradar ${ }^{1, a)}$ \\ ${ }^{1}$ National Physical Laboratory, Dr. K. S. Krishnan Road, New Delhi-110012, India \\ ${ }^{2}$ Instrument Design and Development Center, Indian Institute of Technology Delhi, \\ Hauz, Khas, New Delhi-110016, India
}

(Received 26 July 2008; accepted 20 August 2008; published online 16 September 2008)

\begin{abstract}
Nonvolatile memory effect based on gold nanoparticles (GNPs) doped deformed helix ferroelectric liquid crystal (DHFLC) has been observed. This observed memory effect has been attributed to electric field induced charge transfer from liquid crystal molecules to the GNPs and the stabilization of helix deformation of DHFLC material, which occurs on the application of electric field beyond a critical field. The memory effect has been analyzed by polarizing optical microscopy, high-resolution transmission electron microscopy, and dielectric spectroscopy. The observed memory effect combines two active research areas: nanotechnology and liquid crystal. () 2008 American Institute of Physics. [DOI: 10.1063/1.2980037]
\end{abstract}

The nanoparticles (NPs) doped liquid crystals (LCs) have been studied due to their attractive properties and potential applications in electronic industry by various groups around the world for observing the different aspects such as electro-optical, dielectric, memory effect, phase behavior, etc. ${ }^{1-10}$ Much of the reported work has been focused on nematic liquid crystals (NLCs). ${ }^{2-6,9}$ However, the doping of NPs in ferroelectric liquid crystals (FLCs), which are well known for their good optical contrast, low threshold voltage, memory effect, fast response, etc., is rarely reported in literature. However few studies have been reported on FLCs under the influence of aerosil particles on isotropic-nematic, nematics-smectic $\mathrm{A}$, and smectic A-smectic $\mathrm{C}^{*}$ phase transition. ${ }^{11-14}$

In this series, the enhanced electro-optical properties of gold NPs (GNPs) doped FLCs material have been studied where the interaction of GNPs with LC molecules was taken into account. ${ }^{15}$ The memory effect in filled nematics (nanoparticles or aerosils doped nematics) and NPs doped FLCs have been studied well. ${ }^{15-18}$ The nonvolatile electrical bistability and its mechanism in organic/metal nanoclusters/ organic systems have been demonstrated well. ${ }^{19-21} \mathrm{~A}$ nonvolatile memory system based on GNPs decorated polyaniline nanofibers has been demonstrated by Tseng and co-workers, ${ }^{22,23}$ where the charge transfer effect was taken into account. The deformed helix FLCs (DHFLCs), which is a special kind of FLC, are very useful and have much applications in display devices because of their low driving voltage, gray scale generation capability, easily achievable alignment, fast response, etc. ${ }^{24,25}$ The bistability or memory effect has also been demonstrated by us ${ }^{26-28}$ in DHFLCs.

In this letter, the long nonvolatile memory effect in GNPs doped DHFLC material has been observed which is probably due to the electric field induced charge transfer effect, and the stabilization of helix deformation process. The GNPs were synthesized using citrate reduction method ${ }^{29}$ and characterized by high-resolution transmission electron microscope (HRTEM) (Technai G20- Stwin, USA). For this

\footnotetext{
${ }^{\text {a) }}$ Author to whom correspondence should be addressed. Electronic mail: abiradar@mail.nplindia.ernet.in.
}

study, homogeneously aligned cells (thickness $3 \mu \mathrm{m}$ ) of the DHFLC material (FLC 6304, Rolic, Switzerland) have been prepared. The phase sequence of the material used is as follows:

$$
\text { Cryst. } \stackrel{-14^{\circ} \mathrm{C}}{\leftrightarrow} \mathrm{Sm} \mathrm{C} \mathrm{C}^{*} \stackrel{60.5^{\circ} \mathrm{C}}{\leftrightarrow} \mathrm{Sm} \mathrm{A} \leftrightarrow 4^{\circ} \mathrm{C}
$$

An impedance analyzer (Wayne Kerr, 6540 A, UK) was used to measure dielectric permittivity of the sample in the frequency range of $20 \mathrm{~Hz}-10 \mathrm{MHz}$. The electrical and optical responses were observed by applying electrical pulses of triangular and time delayed square shapes, respectively, to the sample using a storage oscilloscope (HM 1507-3, HAMEG, Germany). The textural micrographs have been taken using polarizing microscope (Ax-40, Carl Zeiss, Germany) and charge coupled device camera.

The size and concentration of GNPs, which were doped in DHFLC, play a critical role for electro-optic response of this material. ${ }^{3}$ Figure 1 shows the distribution of NPs on the scale of $200 \mathrm{~nm}$. The typical size of GNPs prepared was around 15-20 nm. The electrical response of GNPs doped DHFLC material for different frequencies and a fixed voltage on the application of triangular pulse was studied. A symmetric polarization peak was observed in broad frequency range. The electrical response of the pure DHFLC has been re-

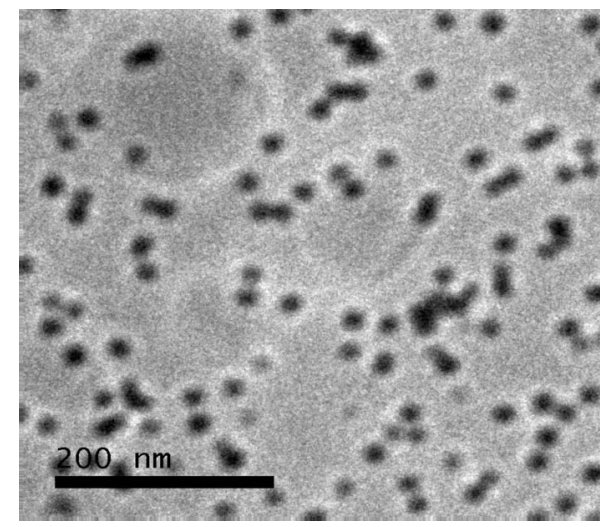

FIG. 1. HRTEM micrograph of synthesized gold nanoparticles. Scale bar: $200 \mathrm{~nm}$. 

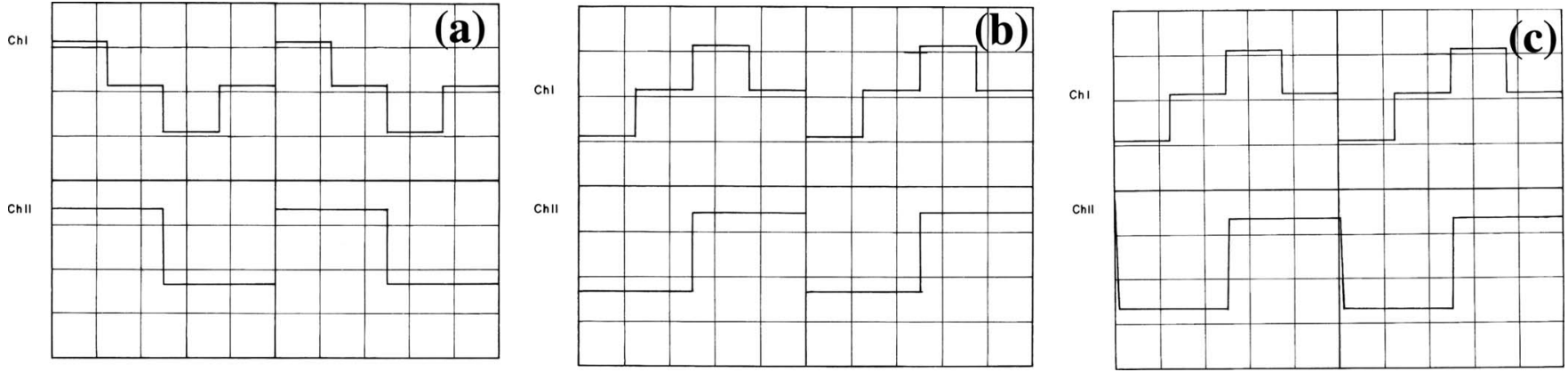

FIG. 2. Optical response of GNPs doped DHFLC at room temperature in $3 \mu \mathrm{m}$ memory cells at $20 \mathrm{~V}$ at (a) $100 \mathrm{mHz}$, (b) $10 \mathrm{~Hz}$, and (c) $100 \mathrm{~Hz}$. CH1 shows driving square voltage and $\mathrm{CH} 2$ shows its optical response. The time scale per division is on $x$-axis, which is same for both channels.

ported elsewhere. ${ }^{28}$ Figure 2 shows that a complete memory effect is observed in the entire frequency range and at a fixed voltage. As seen in the figure the optical transmission changes from maximum to minimum as the applied field reverses its polarity and there is almost no change when the applied field attains its $0 \mathrm{~V}$ state. However, there is slight degradation in the memory at higher frequencies because the memory effect in DHFLC is not favorable at higher frequencies. ${ }^{26}$ The experimental observations by optical response of GNPs doped DHFLC material are found consistent with our earlier observed electrical response of DHFLC material. ${ }^{28}$

It has also been found that the presence of NPs can alter the dielectric behavior dramatically. ${ }^{3-6,30}$ Figure 3 shows the dielectric permittivity $\left(\varepsilon^{\prime}\right)$ as function of frequency in pure and GNPs doped DHFLCs. As can be seen from Fig. 3(a) the change in bias voltage of measuring field from 0 to $8 \mathrm{~V}$, the dielectric permittivity decreases to minimum that is due to the suppression of phason (Goldstone) mode, which occurs due to phase fluctuation of the molecules. ${ }^{31}$ Again on applying $0 \mathrm{~V}$ bias the $\varepsilon^{\prime}$ appears same as it was before applying the bias. This confirms that the cell does not remain in stable state and hence no memory effect. However, in GNPs doped DHFLCs the cell remains in memory state up to some extent that has been reflected in Fig. 3(b). The memory effect in DHFLC is frequency and voltage dependent. ${ }^{26}$ Otherwise the cell of GNPs doped DHFLC would have been remained in perfect stable state.

The electro-optical textures also reveal similar information confirming the above results. Figure 4 shows that GNPs doped DHFLC exhibit clearly the memory effect. Figure 4(a) shows the scattering state before any bias application to cell. A complete switched state was achieved on the application of $15 \mathrm{~V}$ bias [Fig. 4(b)]. Figure 4(c) shows that the memory was retained even after the removal of bias. Even after a time gap of $6 \mathrm{~h}$ the last memory state remains the same [Fig. 4(c)]. Once the cell switches, it tends to remain in that state for a prolonged period of time (for many days). The memory state in GNPs doped DHFLC samples was switched back forcibly to the original state (scattered state) by the application of sinusoidal field of low amplitude $(1 \mathrm{~V})$ and high frequency $(30-50 \mathrm{~Hz})$, which has been shown in Fig. 4(d).

The observation of this long lasting memory can be attributed to the electric field induced charge transfer effect, which has been discussed earlier, ${ }^{19-23}$ for organic/metal/ organic system and GNPs decorated polyaniline fiber systems. When the bias is applied to the sample cells the electrons that reside on the constituents (for example, hydrogen, oxygen, carbon, nitrogen, etc.) of the LC system may gain enough energy to surmount the interface between LC and GNPs and move on to the GNPs against the applied electric field. So the GNPs become more negatively charged whereas
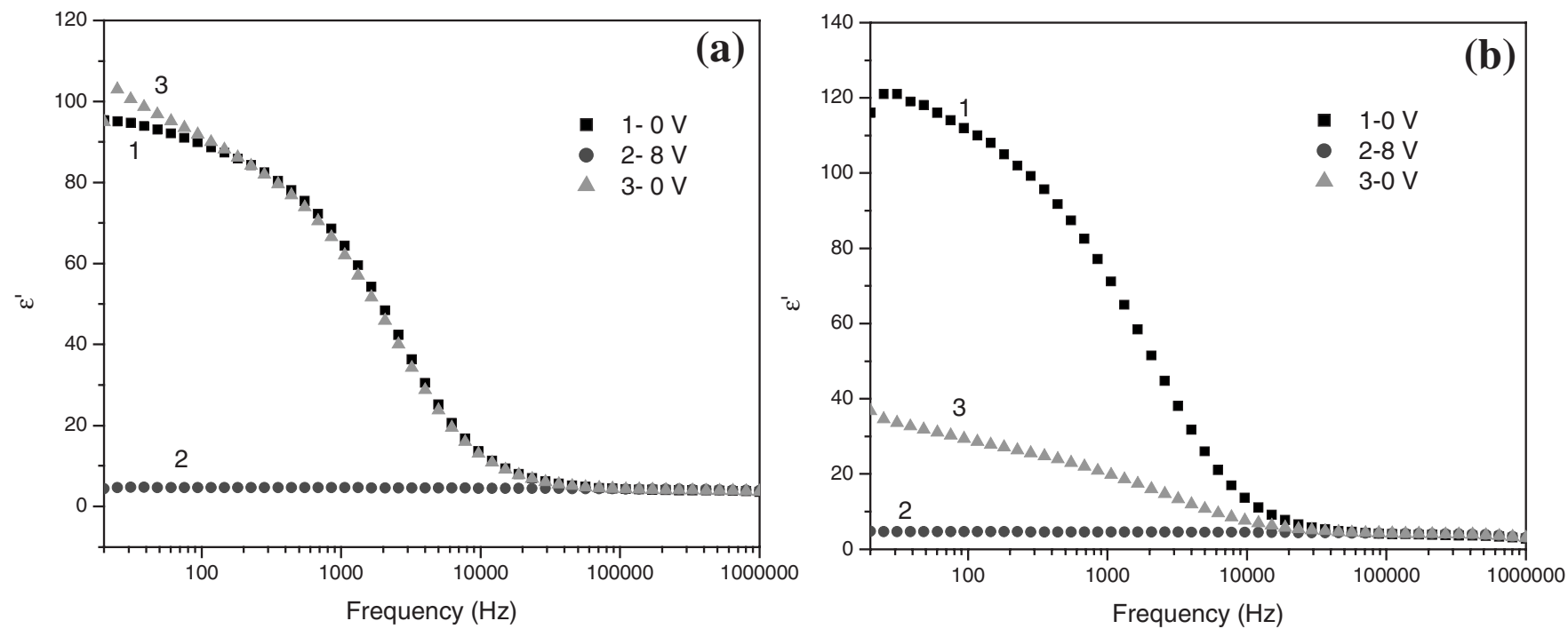

FIG. 3. Dielectric permittivity $\left(\varepsilon^{\prime}\right)$ as a function of $\log$ of frequency $(\log \nu)$ at room temperature at $0 \mathrm{~V}$ (curve 1$), 8 \mathrm{~V}$ (curve 2 ), and again $0 \mathrm{~V}$ (curve 3 ) of (a) pure DHFLC cell of thickness $3 \mu \mathrm{m}$ and (b) GNPs doped DHFLC cell of the same thickness. 

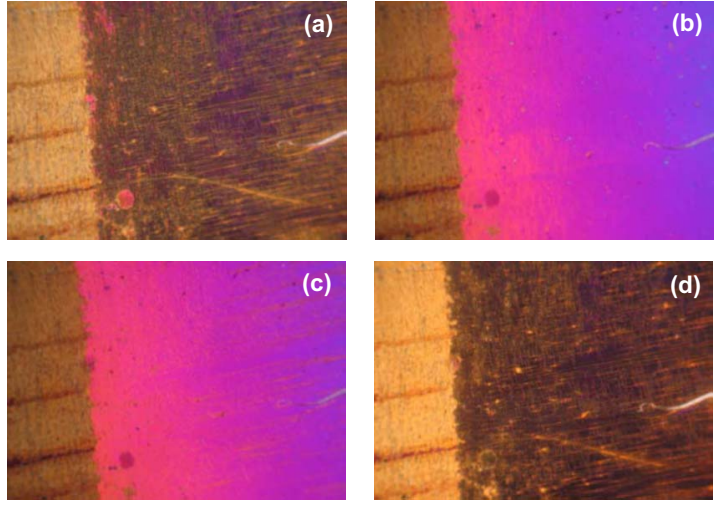

FIG. 4. (Color online) Optical micrographs of $3 \mu \mathrm{m}$ GNPs doped DHFLC memory cell at room temperature at (a) $0 \mathrm{~V}$, (b) $15 \mathrm{~V}$ bias, (c) $6 \mathrm{~h}$ after removal of bias, and (d) again $0 \mathrm{~V}$.

LC more positively charged and hence the switching takes place from off state to on state or dark/bright to bright/dark state. Subsequently the charge is stored around the NPs. This stored charge around the NPs enables the adjacent LC molecules to undergo a change in their properties. Hence the cell undergoes to a switched (changed) state upon the application of the bias field. When the bias is removed the polarized charges cannot recombine and they remain around the NPs. This causes the memory state, i.e., the cell remains in the last or switched state even when the bias is removed.

It has been found that the characteristics of memory effect depend on the chemical nature and properties of both the doped GNPs and LC molecules. ${ }^{18}$ So above phenomenon is not only responsible for the long lasting memory effect, but the properties of DHFLC also play an important role in stabilizing the memory state. It was the stabilization of helix deformation that causes memory effect for a prolonged time as discussed in our previous report ${ }^{28}$ where the pure DHFLC was taken into account. The deformation of helix occurs on the application of electric field perpendicular to helical axis resulting in a rotation of optical axis away from helical axis in a plane perpendicular to the plates. The deformation of helix is due to the interaction of molecular polarization with applied electric field. ${ }^{32}$ Low field causes only a deformation of helix, which explains the fast response and the fast relaxation when the field is removed. Above a certain threshold field helix becomes completely unwound. Hence the retention of prolonged memory state was due to mainly two phenomena that are electric field induced charge transfer and stabilization of deformation respectively.

This electrical bistable behavior, which is reversible in nature, led to memory application to store a virtual ' 0 ' and ' 1 ' (off and on states). Furthermore the bistability remains in either state even after the power is turned off making them ideal candidates for nonvolatile memory devices.

The long lasting memory effect, that may be promising for digital nonvolatile memory devices, has been observed in the GNPs doped DHFLC material. This observation of memory effect has been attributed to the electric field induced charge transfer and stabilization of helix deformation of DHFLC, respectively. The memory effect has been observed for a prolonged time (several days). The promising application of this electrical bistability can be in personal computers, personal digital assistants, digital cameras, etc.
We hope that the bistable devices based on underlying phenomena would have a tremendous impact on the future of information technology and electronic industry. The GNPs doped DHFLC memory system would have high potential for low cost, large area, high speed, high density memory needed for future advance computers and digital electronics.

The authors sincerely thank Dr. Vikram Kumar, Director, National Physical Laboratory, for continuous encouragement and interest in this work. We sincerely thank Dr. S. S. Bawa, Dr. Indrani Coondoo, and Ms. Anu Malik for fruitful discussions. We are also thankful to Dr. Vidyanand Singh, IIT Delhi for helping us in scanning HRTEM images. The authors (J.P. and A.C.) are thankful to CSIR, New Delhi and A.K. to UGC, New Delhi for financial assistance.

${ }^{1}$ S. Zakrevska, Yu. Zakrevskyy, A. Nych, O. Yaroshchuk, and U. Maschke, Mol. Cryst. Liq. Cryst. Sci. Technol., Sect. A 375, 467 (2002).

${ }^{2}$ L. O. Dolgoc and O. V. Yaroshchuk, Colloid Polym. Sci. 282, 1403 (2004).

${ }^{3}$ Y. Reznikov, O. Buchnev, O. Tereshchenko, V. Reshetnyak, A. Glushchenko, and J. West, Appl. Phys. Lett. 82, 1917 (2003).

${ }^{4}$ E. Ouskova, O. Buchnev, V. Reshetnyak, Y. Reznikov, and H. Kresse, Liq. Cryst. 30, 1 (2003)

${ }^{5}$ O. Buchnev, E. Ouskova, Y. Reznikov, V. Reshetnyak, H. Kresse, and A Grabar, Mol. Cryst. Liq. Cryst. 422, 47 (2004).

${ }^{6}$ S. K. Prasad, K. L. Sandhya, G. G. Nair, U. S. Hiremath, C. V. Yelamaggad, and S. Sampath, Liq. Cryst. 33, 1121 (2006).

${ }^{7}$ M. Mitov, C. Bourgerette, and F. de Guerville, J. Phys.: Condens. Matter 16, S1981 (2004).

${ }^{8}$ C. D. Cruz, O. Sandre, and V. Cabuil, J. Phys. Chem. B 109, 14292 (2005).

${ }^{9}$ H. Qi and T. Hegmann, J. Mater. Chem. 16, 4197 (2006).

${ }^{10}$ H. Yoshikawa, K. Maeda, Y. shiraishi, J. Xu, H. Shiraki, N. Toshima, and S. Kobayashi, Jpn. J. Appl. Phys., Part 2 41, L1315 (2002).

${ }^{11}$ M. Marinelli, A. K. Ghosh, and F. Mercuri, Phys. Rev. E 63, 061713 (2001).

${ }^{12}$ A. Hourri, T. K. Bose, and J. Thoen, Phys. Rev. E 63, 051702 (2001).

${ }^{13}$ C. C. Retsch and I. McNulty, Phys. Rev. E 65, 032701 (2002).

${ }^{14}$ S. A. Rozanski and J. Thoen, Ferroelectrics 344, 63 (2006).

${ }^{15}$ S. Kaur, S. P. Singh, A. M. Biradar, A. Choudhary, and K. Sreenivas, Appl. Phys. Lett. 91, 023120 (2007).

${ }^{16}$ M. Kreuzer, T. Tschudi, and R. Eidenschink, Mol. Cryst. Liq. Cryst. Sci. Technol., Sect. A 223, 219 (1992).

${ }^{17}$ R. Eidenschink and W. H. De Jeu, Electron. Lett. 27, 1195 (1991).

${ }^{18}$ A. Glushchenko, H. Kresse, V. Reshetnyak, Y. Reznikov, and O. Yaroshchuk, Liq. Cryst. 23, 241 (1997).

${ }^{19}$ L. P. Ma, J. Liu, and Y. Yang, Appl. Phys. Lett. 80, 2997 (2002).

${ }^{20}$ L. P. Ma, S. Pyo, J. Ouyang, Q. Xu, and Y. Yang, Appl. Phys. Lett. 82, 1419 (2003).

${ }^{21}$ L. D. Bozano, B. W. Kean, V. R. Deline, J. R. Salem, and J. C. Scott, Appl. Phys. Lett. 84, 607 (2004).

${ }^{22}$ R. J. Tseng, J. Huang, J. Ouyang, R. B. Kaner, and Y. Yang, Nano Lett. 5, 1077 (2005)

${ }^{23}$ R. J. Tseng, C. O. Baker, B. Shedd, J. Huang, R. B. Kaner, J. Ouyang, and Y. Yang, Appl. Phys. Lett. 90, 053101 (2007)

${ }^{24}$ L. A. Beresnev, V. G. Chigrinov, D. I. Dergachev, E. P. Poshidaev, J. Funfschilling, and M. Schadt, Liq. Cryst. 5, 1171 (1989).

${ }^{25}$ J. Funfschilling and M. Schadt, J. Appl. Phys. 66, 3877 (1989).

${ }^{26}$ S. Kaur, A. K. Thakur, R. Chauhan, S. S. Bawa, and A. M. Biradar, J. Appl. Phys. 96, 2547 (2004).

${ }^{27}$ A. K. Thakur, S. Kaur, S. S. Bawa, and A. M. Biradar, Appl. Opt. 43 5614 (2004).

${ }^{28}$ J. Prakash, D. S. Mehta, A. Choudhary, S. Kaur, V. Rathore, and A. M. Biradar, J. Appl. Phys. 103, 044103 (2008).

${ }^{29}$ J. Turkevich, P. C. Stevenson, and J. Hillier, Discuss. Faraday Soc. 11, 55 (1951).

${ }^{30}$ L. Cseh and G. H. Mehl, J. Mater. Chem. 17, 311 (2007).

${ }^{31}$ S. Hiller, A. M. Biradar, and W. Haase, Phys. Rev. E 53, 641 (1996).

${ }^{32}$ G. B. Cohen, R. Progreb, K. Vinokur, and D. Davidov, Appl. Opt. 36, 455 (1997) 